\title{
AN INTEGRATED ACCELEROMETER AS A DEMONSTRATION OF A NEW TECHNOLOGY USING SILICON FUSION BONDING AND DEEP REACTIVE ION ETCHING
}

\author{
J. Mohan, ${ }^{2}$ N. I. Maluf, ${ }^{1,2}$ K. E. Petersen, ${ }^{2,3}$ and G. T. A. Kovacs ${ }^{2}$ \\ 'Lucas NovaSensor, 1055 Mission Court, \\ Fremont, CA 94539 \\ ${ }^{2}$ Stanford University, Center for Integrated Systems, \\ Stanford, CA 94305-4075 \\ ${ }^{3}$ Cepheid, 3655 Valley Ridge Lane, \\ San Jose, CA 95148
}

\section{ABSTRACT}

An all single crystal silicon accelerometer fabricated using silicon fusion bonding (SFB) and deep reactive ion etching (DRIE) is presented. The device integrates an interdigitated micromechanical sense element with simple CMOS circuitry. The capacitive sense plates are $60 \mu \mathrm{m}$ deep with an aspect ratio of 15 to 1 . Preliminary testing has shown that large changes in capacitance (> $100 \mathrm{fF}$ ) are achievable. A sensitivity of $90 \mathrm{mV} / \mathrm{g}$ was demonstrated using off-chip commercially available IC's.

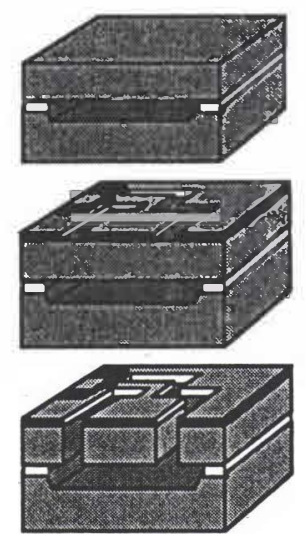

Cavity Etch, Silicon Fusion Bond, Grind \& Polish

CMOS Processing

DRIE Etch Into Cavity

Figure 1: A simplified schematic illustrating the major process steps.

\section{INTRODUCTION}

A new process was recently presented[1] combining silicon fusion bonding (SFB) and deep reactive ion etching (DRIE) for the fabrication of high aspect ratio, single crystal silicon microstructures. It combines the many advantages of "surface" micromachining, namely design flexibility and compatibility with conventional IC fabrication processes, with those of traditional "bulk" micromachining, namely robustness and three-dimensional shaping. The process involves two major steps for the fabrication of the mechanical microstructures: a shallow etch and silicon fusion bonding are used at the beginning to form an embedded cavity, and a deep reactive ion etch down to the cavity is used at the end to release the microstructures. Signal processing circuitry is fabricated using conventional IC fabrication processes between the SFB and DRIE steps (Fig. 1). To demonstrate the capabilities of the SFB/DRIE technology, an accelerometer was fabricated integrating a mechanical sense element with simple signal processing circuitry.

\section{DESCRIPTION}

The interdigitated micromechanical sense element (Fig. 2) takes advantage of the fact that beams fabricated using the above process are compliant in the plane of the die but very stiff in perpendicular directions (Fig. 3) thus minimizing cross-axis sensitivity. Furthermore, the deep etching allows the fabrication of substantially larger proof masses and capacitances than possible using surface micromachining processes. The device typically uses four sets of interdigitated plates, two configured as a capacitive half-bridge for sensing the displacement, and two for electrostatic actuation (Fig. 4). Separating the sense plates from the actuation plates allows large sense signals to be used and thus improves the noise performance for

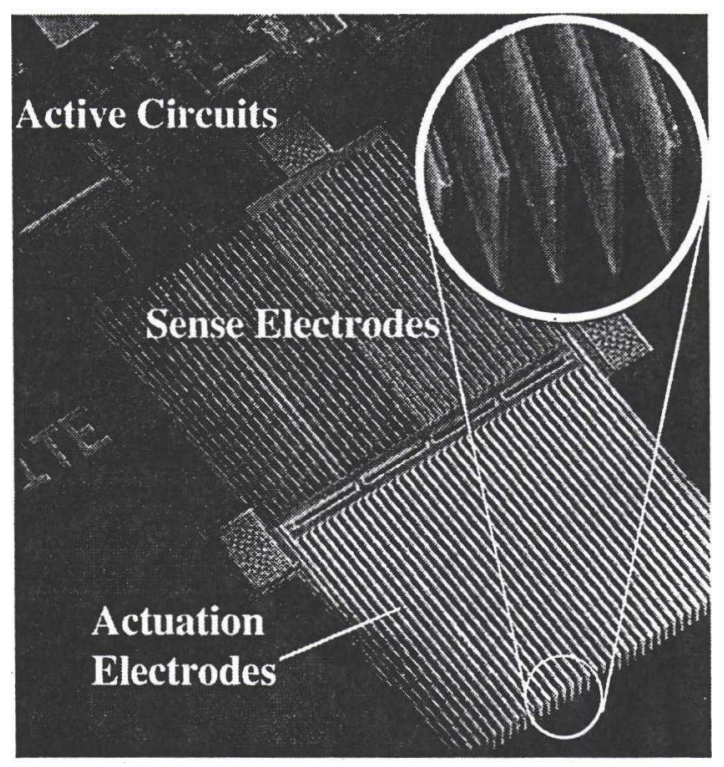

Figure 2: A scanning electron micrograph of a cleaved device showing the interdigitated sense element and part of the surrounding circuitry. The inset shows a magnified image of the high aspect ratio plates. The micromechanical element is $1.5 \times 1.0 \mathrm{~mm}^{2}$ with an overall capacitance of $\sim 6 \mathrm{pF}$.

closed loop. The beams were all $6 \mu \mathrm{m}$ wide with a spacing of $4 \mu \mathrm{m}$ and a depth of $60 \mu \mathrm{m}$. The overall sense capacitance ranges between 2 to $6 \mathrm{pF}$ depending on the size of the element with a change in capacitance per unit of acceleration ranging between 30 and $150 \mathrm{fF} /$ g. Due to the large proof masses (30 to $60 \mu \mathrm{g}$ ), the resonant frequency was less than $3 \mathrm{kHz}$. 


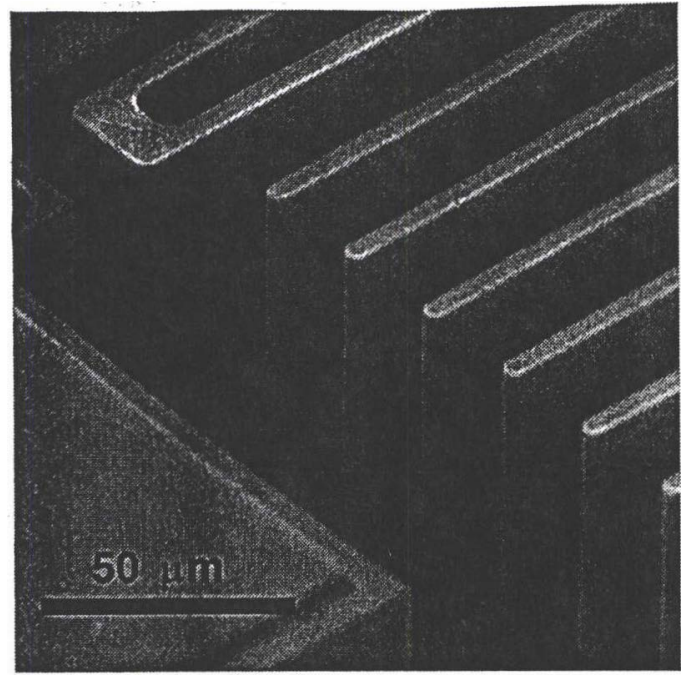

Figure 3: A scanning electron micrograph showing the set of plates attached to the shuttle plate and the edge of the supporting spring. The plates are compliant only in the plane of the die.

\section{RESULTS}

Sense and amplification circuitry was designed and integrated with the micromechanical structures using the Stanford p-well CMOS process. Various building blocks including amplifiers, buffers and a simple demodulator were tested and found to be functional. Due to the large available capacitance, preliminary testing was completed using external circuitry with the accelerometer in open loop. A 100 $\mathrm{kHz}, 40 \mathrm{mV}$ p-p signal was applied to the capacitive half-bridge and the current imbalance was amplified by means of an I-V converter (Linear Technology LT1056) and rectified using a synchronous demodulator (Analog Devices AD630). The accelerometer was mounted alongside a reference accelerometer (for calibration purposes) on a shaker motor and the signals from both devices were recorded. The results, shown in Fig. 5, indicate a sensitivity of $90 \mathrm{mV} / \mathrm{g}$. A minimum resolution of better than $50 \mathrm{mg}$ was measured. This figure is determined by the electronic interface rather than the mechanical performance of the sense element. Mechanical noise figures are estimated at less than $0.2 \mathrm{mg} / \sqrt{\mathrm{Hz}}$. Non-idealities, including non-linearity, are currently being characterized and their sources examined. Change in the capacitance of the sense plates as a function of an electrostatic bias applied to the actuation plates was also measured (Fig. 6). As expected, a non-linear behavior was obtained in reasonable agreement with the results of a simple model using parallel plates. Depending on the stiffness of the supporting spring and the number of biased plates, the "collapse" voltage varied between 3.5 and $25 \mathrm{~V}$.

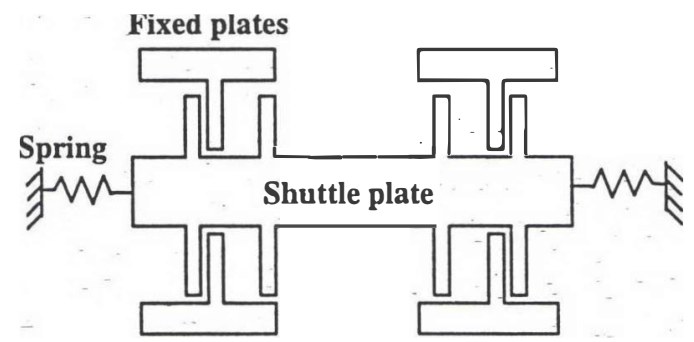

Figure 4: A schematic illustrating the basic mechanical sense element.

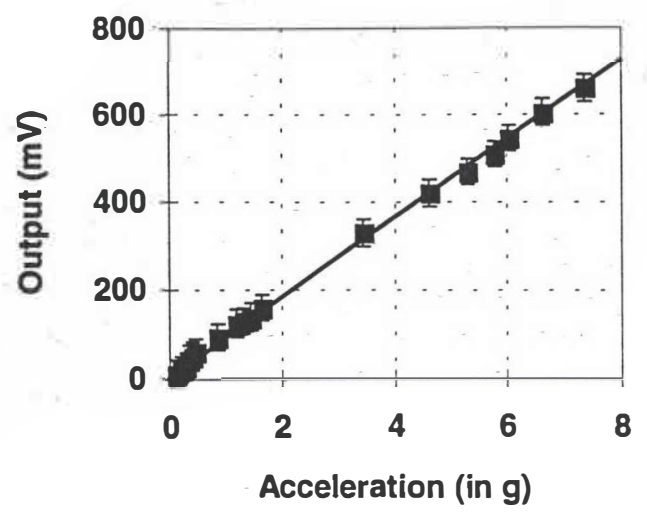

Figure 5: A graph showing the accelerometer measured output voltage as a function of extemally applied acceleration.

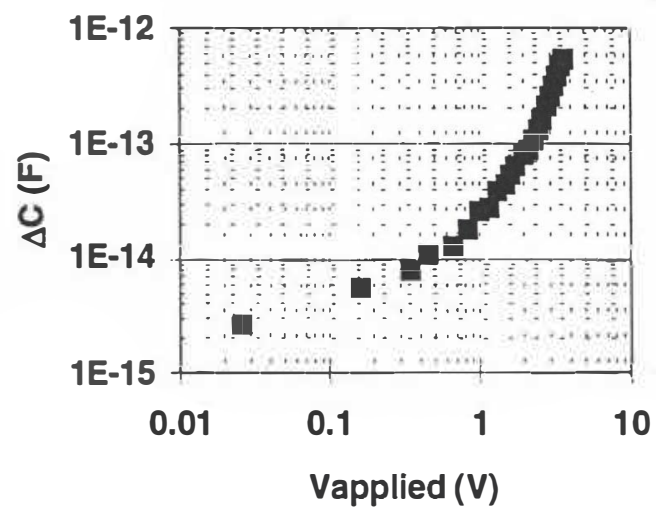

Figure 6: A graph showing change in capacitance as a function of an externally applied electrostatic bias. The base capacitance for this particular device is $3.6 \mathrm{pF}$. The collapse voltage is $>8 \mathrm{~V}$.

\section{SUMMARY}

An accelerometer fabricated using silicon fusion bonding and deep reactive ion etching was presented. The device demonstrates the process capabilities of merging SFB and DRIE for the fabrication of high aspect ratio mechanical microstructures. Ease of integration of signal processing circuitry using conventional CMOS was also demonstrated.

\section{REFERENCES}

1. E. Klaassen, K. Petersen, J. M. Noworolski, J. Logan, N. Maluf, J. Brown, C. Storment, W. McCulley, and G. Kovacs, "Silicon Fusion Bonding and Deep Reactive Ion Etching; A New Technology for Microstructures," in the proceedings of the 8th International Conference on Solid-State Sensors and Actuators, Transducers '95, Stockholm, Sweden, June 1995, p. 556

\section{ACKNOWLEDGMENTS}

This work was funded under DARPA contract DAAL01-94-C3411. We thank C. Storment, A. Partridge, R. Scimeca, E. Klaassen, B. Darling, A. McQuarrie and J. Logan for their assistance. The etching was performed using an STS ICP system. 